\title{
Aerodynamic Performance Analysis of Axial-fan in Low Pressure Pipeline based on ANSYS CFX
}

\author{
Tiancun Yang ${ }^{1, a^{*}}$, Chen Xiaolan ${ }^{1, b}$, Heming Cheng ${ }^{1, c}$ \\ ${ }^{1}$ Mechanical and Electrical Engineering, Huanggang Normal College, Huanggang 438000, China

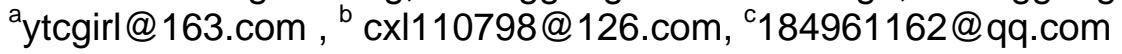

Keywords: ANSYS, CFX, Axial-fan , Aerodynamic performance.

Abstract. ANSYS CFX software is used to simulate and analyze the axial -fan for heat sending in the pipe tunnel kiln, and find the relationship between the total pressure and the total pressure efficiency of the fan at different blade angles and different inlet air flow conditions. According to analyze the effects on axial-fan performance, identify best working area of the fan. This work can play a guiding role for the designers in reducing the efficiency loss of fans, and also make a contribution to energy savings from product design point.

\section{Introduction}

Axial-fan named after the fluid flows into impeller and out of both along the axial direction, which is the direction of airflow and the impeller shaft in the same direction. In the most common life, electric fans and air conditioning both belong to axial flow run fan [1]. Currently axial-fan performance is not satisfactory which used in the tunnel kiln, no thorough design and calculation, the actual performance of air volume and wind pressure is not good, air volume increase at the expense of pressure drop, sending wind distance is greatly reduced. Thus the drying effect can not reach to standard. This brings out collapse of semi finished brick, even explosion. Because of the special structure and complex internal flow field, the conventional test method is difficult to optimize wind turbine, currently often use finite element method to predict fan aerodynamic performance. In this paper, ANSYS CFX software is used to simulate and calculate the effect of fan performance at different inlet flows and different blade installation angles, to find out the relationship between the total pressure and the total pressure efficiency. Accordingly obtain the best working conditions of axial-fans in the tunnel kiln area.

\section{Establish a geometric model}

Firstly, build axial-fan 3D model, where ignore motor supporting member, the impact of the cooling lubricant tube and motor cables, left only the main structure of the axial-fan. Blades of the fan model adopt for low-speed environment airfoil NACA-64 [2] . Fan parameters are as follows: the airfoil width $180 \mathrm{~mm}$, length $280 \mathrm{~mm}$, the number of blades 10 , the sweeping diameter $880 \mathrm{~mm}$, the cabinet inner diameter $900 \mathrm{~mm}$, chassis length $1960 \mathrm{~mm}$, inlet diameter $1800 \mathrm{~mm}$, the outlet diameter $900 \mathrm{~mm}$. According to the traditional design experience, chose $30^{\circ}, 34^{\circ}, 38^{\circ}, 42^{\circ}, 46^{\circ}$ five typical blade installation angles.

Modeling of wind turbine blades belongs to complicated surface modeling, direct modeling is not feasible, so only divide the blade into several cross-section, calculate the coordinates of boundary 
points of each section, and then use the coordinates to generate curves and surfaces. Axial-fan blades and frame model is shown as Fig. 1

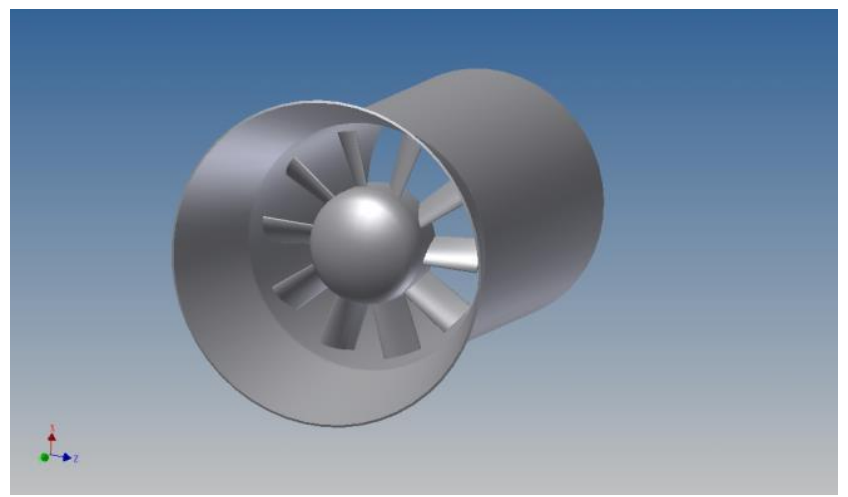

Fig.1 Axial-fan 3D model

\section{CFX calculation}

Deal with the flow field of axial-fan 3D model, then import in CFX. In order to avoid the cumbersome conventional flow field and boundary conditions setting step, we use the ANSYS Workbench software to calculate, friendly interface, you can quickly link between each module and automatically complete the modeling and solving work. Modeling, simulation, analysis, pretreatment and after handling achieve seamlessly connection.

Use ICEM CFD software to part structured grid, after optimization, get a total of 342,226 nodes, $1,812,561$ high-precision units, units quantity and quality can meet the requirement of the next calculation. Blades and hub calculation mesh shown in Fig 2.

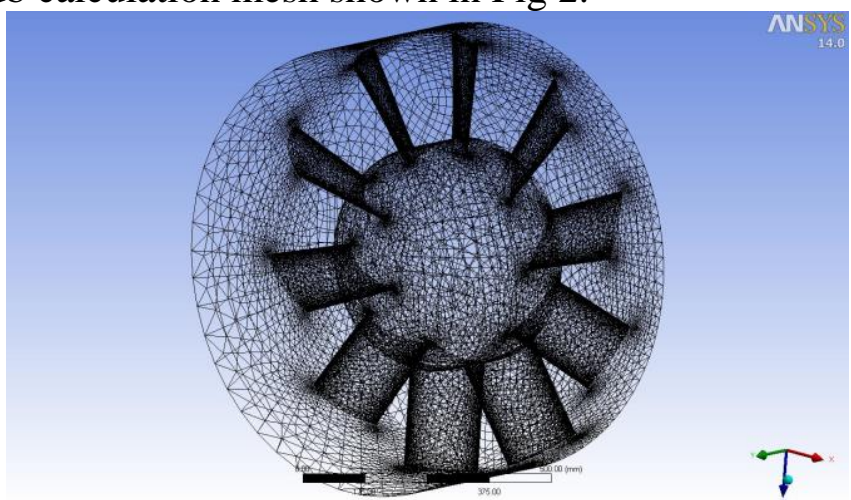

Fig. 2 Blade and Hub Mesh Picture

Use RNG K- $\varepsilon$ two-equation turbulence model to simulate numerical date of axial-fan, pressure-velocity coupling using SIMPLEC algorithm [3] [4] [5].

\subsection{Basic equations}

The standard $k-\varepsilon$ model need to solve the turbulent kinetic energy and dissipation rate equation. Turbulent kinetic energy transport equation is derived through a precise equation, but the dissipation rate equation is by physical reasoning, mathematical simulation similar to the prototype of the equation. This model assumes that the flow is fully turbulent, viscous molecule can be ignored. Therefore, the standard model is only suitable for fully turbulent flow simulation.

In standard $k-\varepsilon$ model, turbulent kinetic energy $k$ (2-1) and the dissipation rate $\varepsilon$ (2-2) equation as follows:

$$
\begin{aligned}
& \rho \frac{D k}{D t}=\frac{\partial}{\partial x_{i}}\left[\left(\mu+\frac{\mu_{t}}{\sigma_{k}}\right) \frac{\partial k}{\partial x_{i}}\right]+G_{k}+G_{b}-\rho \varepsilon-Y_{M} 2-1 \\
& \rho \frac{D \varepsilon}{D t}=\frac{\partial}{\partial x_{i}}\left[\left(\mu+\frac{\mu_{t}}{\sigma_{k}}\right) \frac{\partial \varepsilon}{\partial x_{i}}\right]+C_{1 \varepsilon} \frac{\varepsilon}{k}\left(G_{k}+C_{3 \varepsilon} G_{b}\right)-C_{2 \varepsilon} \rho \frac{\varepsilon^{2}}{k} 2-2
\end{aligned}
$$


In the above equation, $G_{k}$ represents turbulent kinetic energy which generated by the average velocity gradient, $G_{b}$ represents turbulent kinetic energy which caused by buoyancy influences; $Y_{M}$ is the impact compressible turbulence expansion to the total dissipation rate. Turbulent viscosity coefficient is $\mu_{t}=\rho C_{\mu} \frac{k^{2}}{\varepsilon}$ 。

In the RNG K- $\varepsilon$ model , transmission model of turbulence generation and dissipation is the same as the $k-\varepsilon$ model, but the model constant is not the same. The original set $C_{1}$ is replaced by the function $\mathrm{C}_{1 \mathrm{RNG}}$. Equation (2-3) the following form:

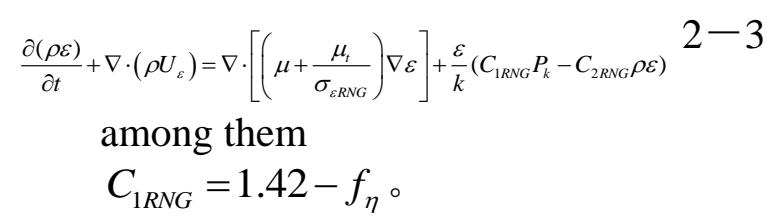

\subsection{Boundary conditions set}

FEM Essence is solving differential equations, differential equations have a definite solution, it must be introduced into the conditions, these additional conditions called boundary conditions. In the form of many boundary conditions, the most common two - the initial onditions and boundary conditions.

Axial-fan structure is divided into motive areas and still areas, impeller and hub belong to the motive area, using a rotary coordinate. other regional stationary using fixed coordinates. Impeller rotation speed is defined as 1450rpm, still areas 0rpm, the two regions through interface connection. Setting the airflow inlet boundary condition is converted air flow to mass . Then will define a set of flow rate to weigh the fan performance, taking into account real factory parameters, the flow rate range is set from $40000 \mathrm{~m}^{3} / \mathrm{h}$ to $60000 \mathrm{~m}^{3} / \mathrm{h}$ between.

\section{Analysis}

The main criterion of axial-fan performance are: flow rate, total pressure and total pressure efficiency (excluding motor efficiency), here total pressure of axial-fans is import and export pressure difference. And under different inlet air flow conditions, the relationship between the total pressure and the total pressure efficiency between fans of this paper calculate different blade angles. Figure 3 and Figure 4 is at blade angle $38^{\circ}$, flow rate $45000 \mathrm{~m}^{3} / \mathrm{h}$, air flow velocity inside the encasement and overall pressure cloud; Fig. 5 the blade installed at five different angles, inlet flow and total pressure comparison; Fig. 6 the blade installed in five different angles, the fan flow and total pressure efficiency comparison chart.

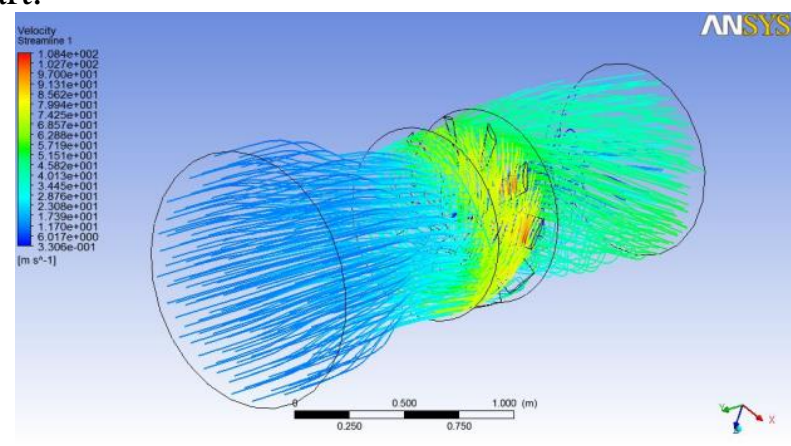

Fig. 3 Air Flow Velocity inside the Encasement 


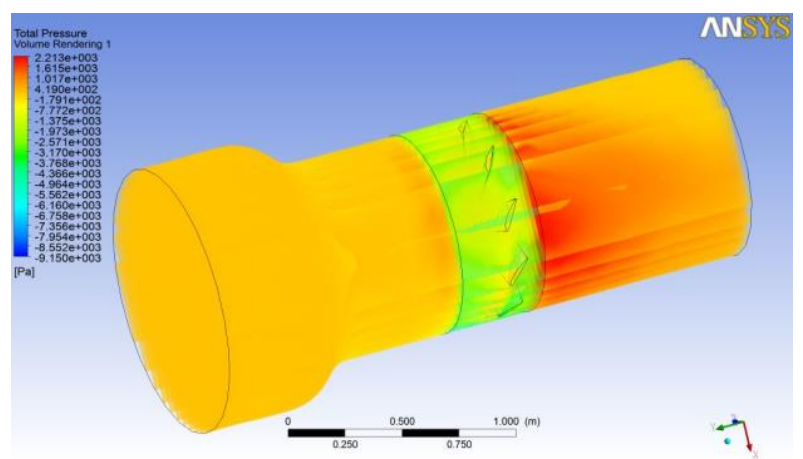

Fig. 4 Fan Overall Pressure Cloud

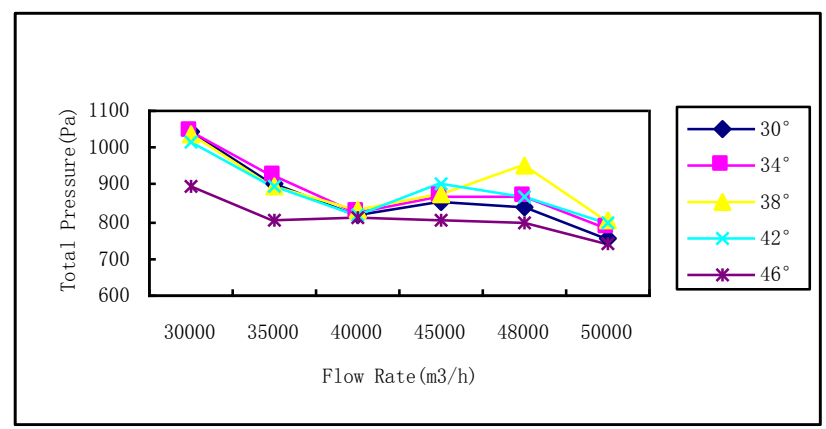

Fig. 5 Flow rate-Total Pressure Comparison Chart

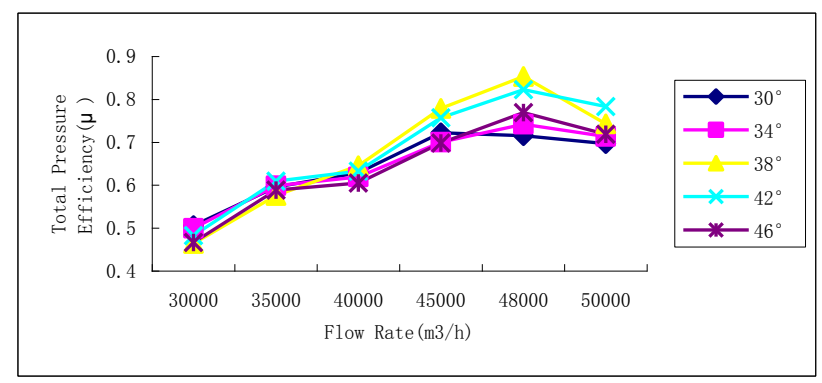

Fig. 6 Flow rate-Total Pressure Efficiency Comparison Chart

It is clear from Fig. 5, when the flow rate is less than $40000 \mathrm{~m}^{3} / \mathrm{h}$, as the flow rate increases, the total pressure decreases, after more than $40000 \mathrm{~m}^{3} / \mathrm{h}$, the total pressure will be increased as the flow rate increases and then decreases changes; Fig.6, as the flow increases, the fan total pressure efficiency curve is first increased and then decreased, the highest point of inflection is the efficiency, then the ratio of the lowest energy consumption.

To sum up, at blade angle $38^{\circ}$, the flow rate $48000 \mathrm{~m}^{3} / \mathrm{h}$, comprehensive aerodynamic performance parameters is the best. At the same time, we can also see that when flow rate is less than $40000 \mathrm{~m}^{3} / \mathrm{h}$, the total pressure is large, and the total pressure efficiency is very low. This is because the flow is too small, the entrance will produce negative pressure, resulting in a positive pressure at the exit value is not high, although the higher value of the total pressure, but the low total pressure efficiency, this condition is undesirable.

\section{Conclusion}

According to above simulation analysis, the best work area of the axial-fan is near blade mounting angle at $38^{\circ}$, flow rate $48000 \mathrm{~m}^{3} / \mathrm{h}$. The next step is to contact the manufacturer prototype 
trial to validate simulation results. You can get tunnel kiln axial-fan key performance parameters based on the simulation results, and then provide guidance for the design direction, to avoid more serious design errors and waste of resources, reduce test cost, thus improving research and tunnel kiln axial-fan design level.

\section{Reference}

[1] Wu Yulin, Chen Qingguang, Liu ShuHong, Fan and Compressor [M] Beijing: Tsinghua University Press, 2006

[2] Zhang YuCheng, Yi dengli, Feng Dianyi, Fan Design and Selection [M] Beijing: Chemical Industry Press, 2011

[3] Tang Xuein, Jia Yuxia, Wang Fujun etc. Numerical Simulation Value of Hydrofoil Cavitation

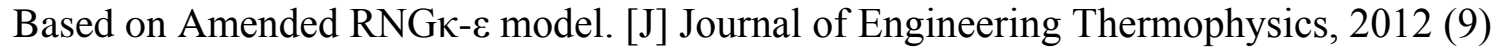

[4] Wei Lina, Wang Yanfei, Automotive Centrifugal Pump Performance Analysis Based on CFD [J]. Changchun University of Technology (Natural Science Virson), 2014 (5)

[5] Zhang Xi, Based on CFD Structure Optimization with Axial-Fan in Air Conditioning [D]. Donghua University,2007 